Check for updates

Cite this: Chem. Commun., 2021, 57,7406

Received 17th May 2021

Accepted 14th June 2021

DOI: $10.1039 / \mathrm{d} 1 \mathrm{cc} 02587 \mathrm{~b}$

rsc.li/chemcomm

\section{Accessing novel fluorinated heterocycles with the hypervalent fluoroiodane reagent by solution and mechanochemical synthesis $\dagger$}

\author{
William Riley, ${ }^{a}$ Andrew C. Jones, (D) ${ }^{b}$ Kuldip Singh, ${ }^{a}$ Duncan L. Browne (D) ${ }^{c}$ and \\ Alison M. Stuart (D) *a
}

\begin{abstract}
A new and efficient strategy for the rapid formation of novel fluorinated tetrahydropyridazines and dihydrooxazines has been developed by fluorocyclisation of $\beta, \gamma$-unsaturated hydrazones and oximes with the fluoroiodane reagent. Mechanochemical synthesis delivered fluorinated tetrahydropyridazines in similar excellent yields to conventional solution synthesis, whereas fluorinated dihydrooxazines were prepared in much better yields by ball-milling.
\end{abstract}

Over $85 \%$ of small molecule pharmaceuticals approved by the FDA in 2019 contained a nitrogen-based heterocycle and over a quarter of all FDA approved drug molecules on the market contain at least one fluorine atom. ${ }^{1 a}$ The prominence of heterocycles comes from their activity in vivo, where, specifically their structure provides rigidity to molecular shape and enables effective binding. The pharmaceutical industry is particularly interested in heterocycles with a high $\mathrm{sp}^{3}$ content $^{1 b}$ and the $\mathrm{C}-\mathrm{F}$ bond has a great influence on the pharmacokinetic properties of biologically active molecules. ${ }^{1 c}$

Fluorinated N-heterocycles can be accessed in a single step by intramolecular fluorocyclisations of unsaturated substrates containing an internal nucleophile. The fluorocyclisations of $\beta, \gamma$-unsaturated hydrazones with Selectfluor produced the 5-membered, fluorinated dihydropyrazoles (Scheme 1A). ${ }^{2}$ In one report, fluorinated tetrahydropyridazines were formed along with the dihydropyrazoles when $\mathrm{R}$ was an aryl group. ${ }^{2}$ Similarly, the fluorocyclisations of $\beta, \gamma$-unsaturated oximes formed the 5-membered, fluorinated dihydroisoxazoles using either Selectfluor, ${ }^{3 a, b}$ or hypervalent iodine reagent, $\mathrm{PhI}(\mathrm{OPiv})_{2}$,

\footnotetext{
${ }^{a}$ School of Chemistry, University of Leicester, Leicester, LE1 7RH, UK. E-mail: Alison.Stuart@le.ac.uk

${ }^{b}$ Cardiff Catalysis Institute, School of Chemistry,

Cardiff University, Cardiff, CF10 3AT, UK

${ }^{c}$ School of Pharmacy, UCL, London, WC1N 1AX, UK.

E-mail: Duncan.Browne@ucl.ac.uk

$\dagger$ Electronic supplementary information (ESI) available: Experimental procedures and NMR spectra. CCDC 2083051-2083056. For ESI and crystallographic data in CIF or other electronic format see DOI: $10.1039 / \mathrm{d} 1 \mathrm{cc} 02587 \mathrm{~b}$
}

with HF-pyridine. ${ }^{3 c}$ The synthesis of the 6-membered, fluorinated dihydrooxazines has not been reported (Scheme 1B).

Since its inception, the hypervalent iodine(III) reagent, fluoroiodane 1 (Scheme 1C), has emerged as an excellent fluorinating reagent with a wide range of applications. ${ }^{4,5}$ In many cases, fluoroiodane 1 exhibits differing reactivity to that observed with fluoraza reagents such as Selectfluor. We recently employed fluoroiodane $\mathbf{1}$ in the synthesis of fluorinated lactones using either $\mathrm{AgBF}_{4}{ }^{6 a}$ or hexafluoroisopropanol (HFIP) ${ }^{6 b}$ to activate the fluoroiodane reagent. Looking to further explore the utility of this reagent in fluorocyclisations, we investigated the synthesis of fluorinated tetrahydropyridazines and dihydrooxazines. We also applied ball-milling to the fluorocyclisations since

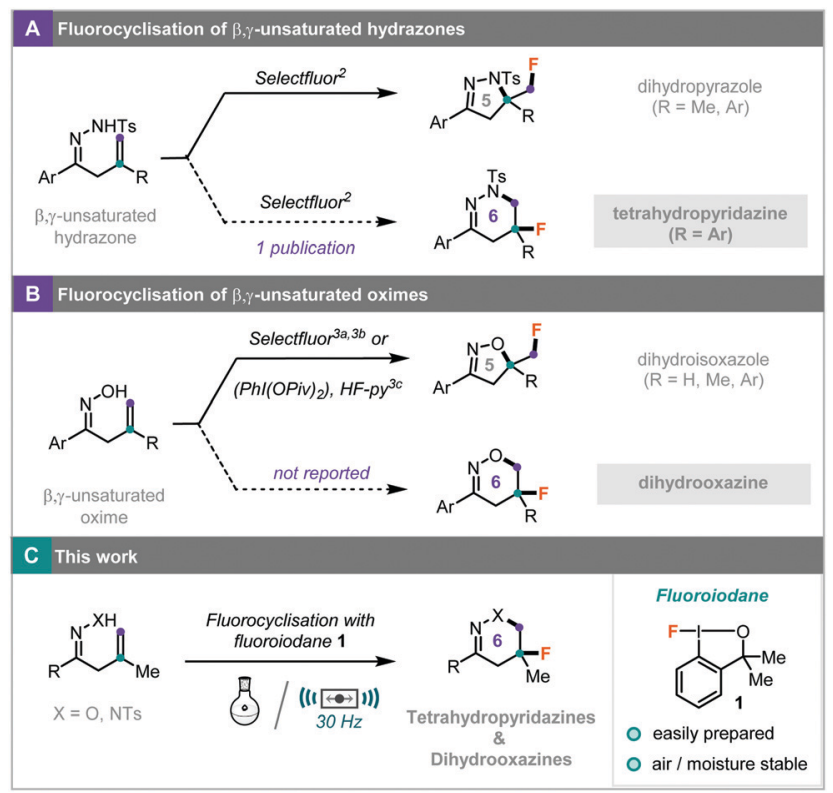

Scheme 1 (A) Synthesis of fluorinated dihydropyrazoles. (B) Synthesis of fluorinated dihydroisoxazoles. (C) Synthesis of fluorinated tetrahydropyridazines and dihydrooxazines. 
Table 1 Optimisation of $\beta, \gamma$-unsaturated hydrazone fluorocyclisation ${ }^{a}$

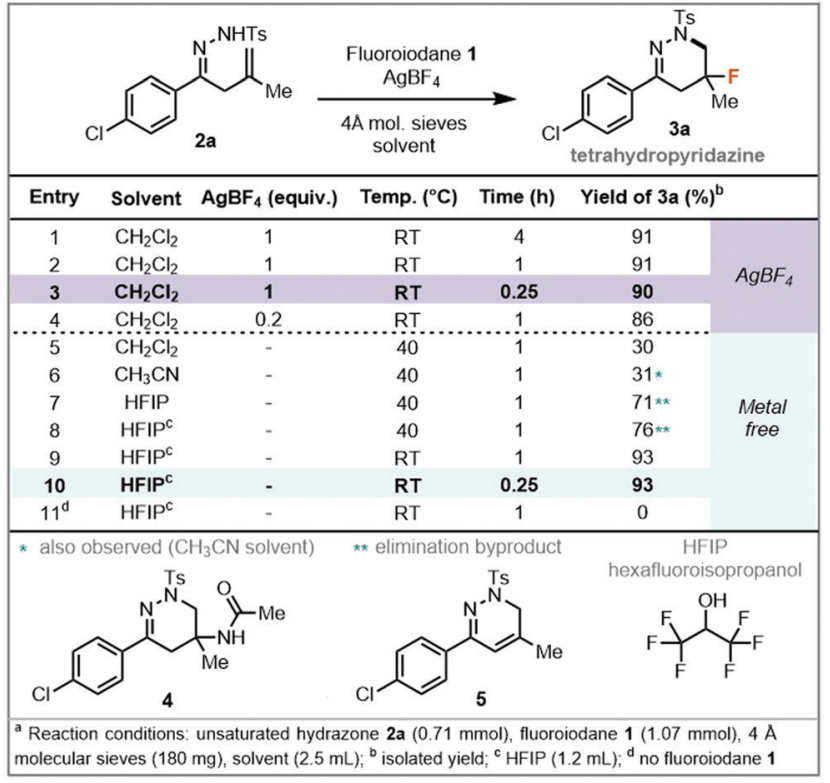

mechanochemical synthesis can minimise solvent requirement and in many cases, can provide enhanced and altered reactivity. ${ }^{7}$ Ball-milling has shown promise in bromocyclisations $^{8}$ and in fluorination reactions, where Selectfluor can selectively mono- or di-fluorinate 1,3-dicarbonyl compounds, ${ }^{9}$ fluorinate pyrazolone rings, ${ }^{10 a}$ and be used in asymmetric organocatalysis. ${ }^{10 b}$ Herein, we report the regioselective synthesis of novel fluorinated 6-membered heterocycles, tetrahydropyridazines and dihydrooxazines, alongside the use of mechanochemistry to minimise halogenated solvents and to improve the yields in the preparation of fluorinated dihydrooxazines.

We began our studies by investigating the fluorocyclisation of hydrazone 2a. To our delight, 2a was fluorocyclised at room temperature in 4 hours using fluoroiodane 1 (1.5 equiv.) with $\mathrm{AgBF}_{4}$ (1 equiv.) in $\mathrm{CH}_{2} \mathrm{Cl}_{2}$ to produce the desired fluorinated tetrahydropyridazine 3a in $91 \%$ yield (Table 1 , entry 1 ). The reaction time could be reduced to just 15 minutes with minimal decrease in yield (entry 3). Alternatively, the amount of $\mathrm{AgBF}_{4}$ could be decreased to 0.2 equivalents in a 1 hour reaction to form the product in $86 \%$ yield (entry 4 ). On further investigation of solvent and temperature (entries 5-10), a set of metalfree conditions were identified using HFIP as the solvent and activator of fluoroiodane $\mathbf{1}$ giving $\mathbf{3 a}$ in $93 \%$ yield after only 15 minutes (entry 10). Notably, whilst use of acetonitrile as solvent did furnish the desired fluorinated product 3a, Ritter product 4 was also formed in $23 \%$ yield suggesting that a tertiary carbocation is a key intermediate in the fluorocyclisation. In entries 7 and 8 where product yield was lower at $40{ }^{\circ} \mathrm{C}$, elimination byproduct 5 was also observed in $10-15 \%$ yield.

Using mechanochemistry the unsaturated hydrazones could efficiently undergo fluorocyclisations with fluoroiodane $\mathbf{1}$ (1.5 equiv.) and just 2 equivalents of HFIP (an 8 fold reduction in the amount of HFIP required, ESI, $\uparrow$ Table S3) to provide an alternative, solvent minimised method. For this protocol,

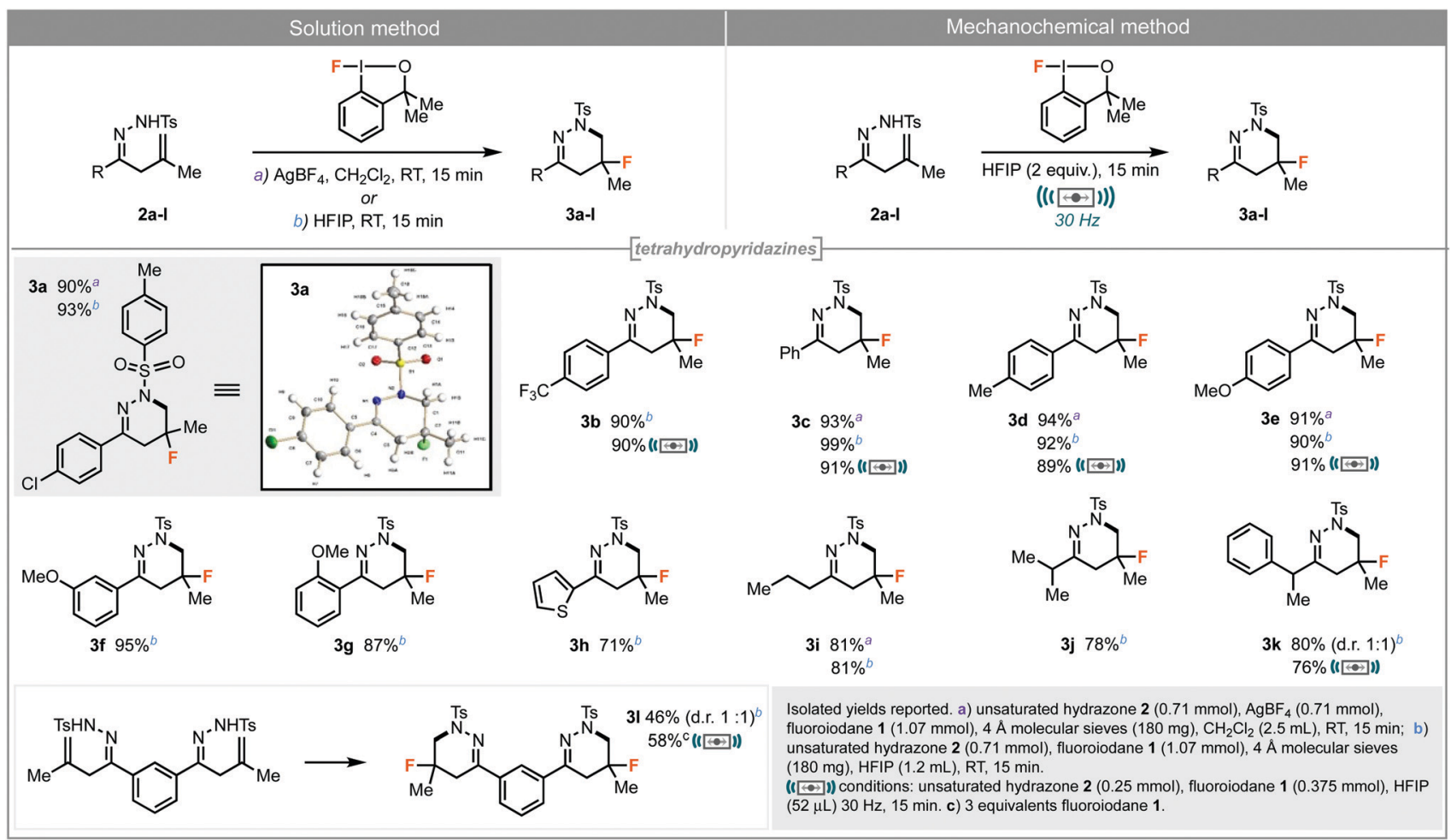

Scheme 2 Scope of 5 -fluorinated tetrahydropyridazine synthesis by solution and mechanochemical synthesis. 
a $10 \mathrm{~mL}$ stainless steel milling jar was used with a $2.5 \mathrm{~g}$ stainless steel milling ball, and the reaction mixture was milled at $30 \mathrm{~Hz}$ for 15 minutes.

With optimal conditions for both a solution and ball-milling method in hand, application to a range of unsaturated hydrazones was explored (Scheme 2). The reaction was tolerant for a series of aryl substituted hydrazones (Scheme 2, 3a-h) with examples of both electron-donating (3e) and electron-withdrawing aryl groups (3b). Although synthesis of the thiophene substituted hydrazone proceeded efficiently (3h), the aromatic byproduct, 5-methyl-3-thiophen-2-yl pyridazine, was also formed in $16 \%$ yield. The fluorocyclisation proved successful on alkyl substituted hydrazones (3i-k) including benzyl and the more hindered isopropyl hydrazones. Bis-tetrahydropyridazine (31) was prepared by a double fluorocyclisation. In all cases, metal-free protocols could be employed and delivered similar yields in comparison to reactions containing $\mathrm{AgBF}_{4}$. The mechanochemical approach was applied to a small range of substrates (3b-e, $\mathbf{3 k}$ and $\mathbf{3} \mathbf{l}$ ) and provided comparable excellent yields to those observed in solution demonstrating an efficient, solvent minimised method. Further studies revealed that catalytic amounts of HFIP ( 0.1 equiv.) activated fluoroiodane $\mathbf{1}$ in $\mathrm{CH}_{2} \mathrm{Cl}_{2}$ and delivered fluorinated tetrahydropyridazine $\mathbf{3 a}$ in $90 \%$ yield (Scheme S1, ESI $\dagger$ ).

Initial results with $\beta, \gamma$-unsaturated oximes demonstrated that they could undergo fluorocyclisation to form dihydrooxazines. This class of fluorinated heterocycle has not been reported previously. After optimisation for traditional solution conditions (ESI, $\uparrow$ Table S4), dihydrooxazine 7a could be formed in $46 \%$ yield after 15 minutes with 1 equivalent of $\mathrm{AgBF}_{4}$ (Table 2A). Notably, dihydroisoxazole 8, intercepted by the benzyl-alcohol backbone of the fluoroiodane reagent, was also isolated as a side product in $15 \%$ yield. In an attempt to improve yield and selectivity towards the desired product, attention was turned to mechanochemical techniques commencing with the conditions used for hydrazone fluorocyclisations (Scheme 2). Fluorocyclisation of unsaturated oxime $\mathbf{6 b}$ using fluoroiodane $\mathbf{1}$ proceeded to give $36 \%$ of the desired product with 2 equivalents of HFIP (Table 2, entry 1). Increased loading of HFIP improved the yield of dihydrooxazine $7 \mathbf{b}$, but also increased the amount of unwanted dihydroisoxazole 9 (entry 2). A small amount of oxazine 10, resulting from elimination, was also observed. Switching to $\mathrm{AgBF}_{4}$ showed decreased activity initially (entry 3 ). However, using $\mathrm{AgBF}_{4}$ with 5 equivalents of $\mathrm{CH}_{2} \mathrm{Cl}_{2}$ provided a dramatic improvement in the yield of dihydrooxazine $7 \mathbf{b}$ ( $80 \%$ isolated yield), and reduced the formation of side products 9 and 10 to 1\% (entry 5). Further studies on the two additives and reaction time revealed that 2 equivalents of $\mathrm{AgBF}_{4}$ and 5 equivalents of $\mathrm{CH}_{2} \mathrm{Cl}_{2}$ in a 1 hour reaction was optimal (entries 6-10). In the absence of HFIP or $\mathrm{AgBF}_{4}$, the reaction showed no activity and returned quantitative amounts of oxime $\mathbf{6 b}$ (entry 11).

A range of $\beta, \gamma$-unsaturated oximes was examined and under traditional solution conditions, aryl unsaturated oximes could undergo fluorocyclisations in moderate yield (Scheme 3A, 7a-7d, 42-53\%). However, applying mechanochemical conditions to
Table 2 Mechanochemical optimisation of $\beta, \gamma$-unsaturated oxime fluorocyclisation
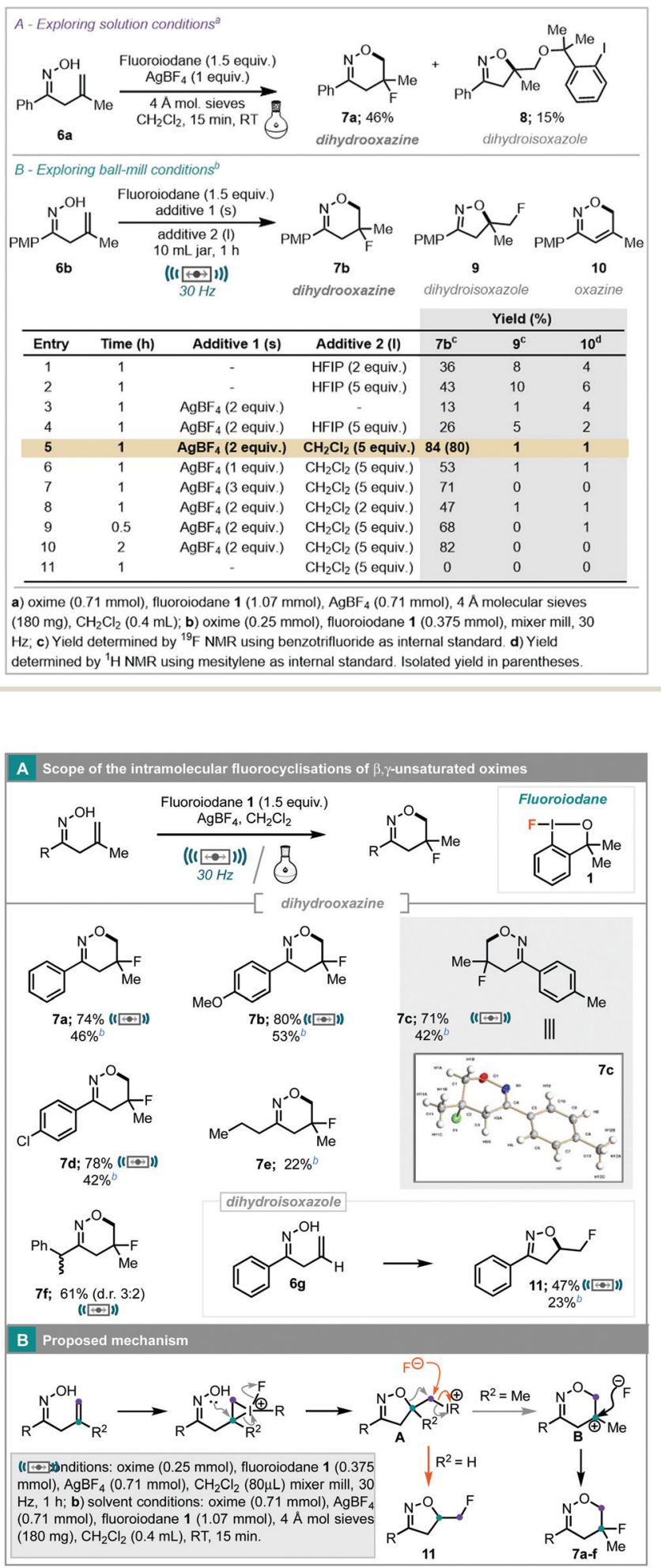

Scheme 3 (A) Scope of 5-fluorodihydrooxazine synthesis. (B) Proposed mechanism.

these substrates greatly improved the yields delivering products $7 \mathbf{a}-7 \mathbf{d}$ in $\mathbf{7 1 - 8 0 \%}$ yield. Alkyl oximes could also be tolerated 


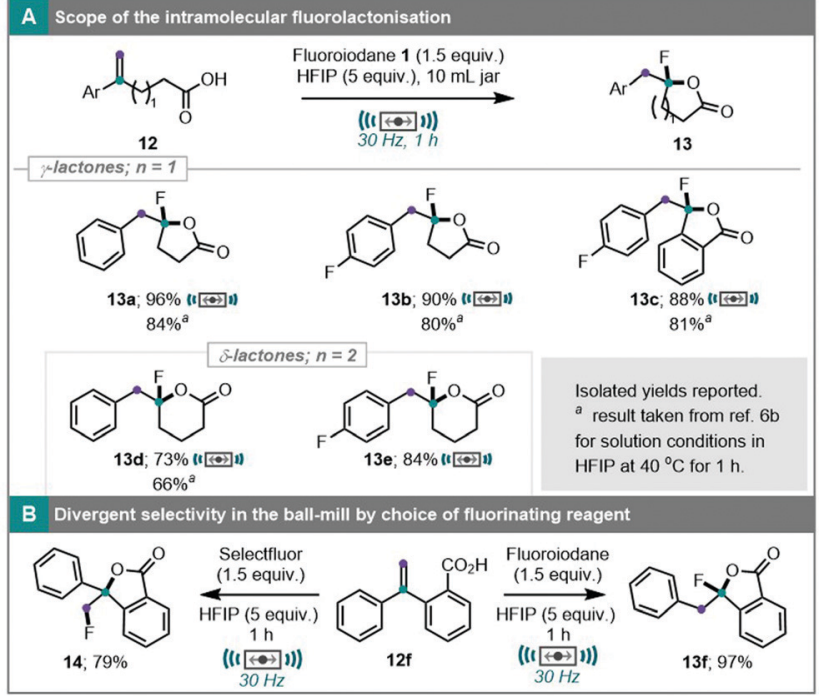

Scheme 4 (A) Scope of ball-milled intramolecular fluorolactonisation. (B) Switching of cyclisation selectivity by choice of fluorinating reagent.

(Scheme 3A, 7e-7f), but oxime 6e gave a lower yield as it contained a $1: 1$ mixture of $(Z)$ - and $(E)$-isomers and only the (Z)-isomer fluorocyclised. Unsaturated oxime $\mathbf{6 g}$ with a mono-substituted alkene selectively formed dihydroisoxazole 11, as reported by Wang using $\mathrm{PhI}(\mathrm{OPiv})_{2}$ and HF.pyridine. ${ }^{3 c}$ This result supports our proposed mechanism (Scheme 3B) where there is initial formation of a 5-membered heterocycle $\mathbf{A}$, followed by ring expansion via tertiary carbocation B, to afford the 6-membered fluorinated heterocycle. However, for mono-substituted unsaturated oximes, formation of a secondary carbocation is not favoured and instead attack of fluoride at the methylene site forms the 5-membered product. Good evidence that tertiary carbocation $\mathbf{B}$ is a key intermediate was provided by the formation of Ritter product $\mathbf{4}$ in the fluorocyclisation of $2 \mathbf{a}$ in acetonitrile (Table 1, entry 6). Hara has also reported a similar ring expansion in the fluorocyclisation of 1-decen-5-ol with iodotoluene difluoride. $^{11,12}$

It is intriguing that a rearrangement, driven by carbocation stability, still occurs under solvent minimised conditions in a ballmill. On a similar thread, we also explored ball-milling techniques in the already established fluoroiodane mediated fluorolactonisation to furnish a range of $\gamma$ - and $\delta$-lactones 13a-f (Scheme 4). ${ }^{6 b}$ These products can only be obtained from a reaction mechanism involving a fluorocyclisation and an aryl migration (Scheme S2, ESI $\dagger) .{ }^{6 a}$ It is remarkable that the aryl migration occurred in the ball-mill with a 6 fold reduction in the volume of HFIP. To demonstrate this further, treatment of unsaturated carboxylic acid $12 f$ with Selectfluor under ball-milling conditions for 1 hour at $30 \mathrm{~Hz}$ furnished fluorolactonised product $\mathbf{1 4}$ without an aryl migration, and so, the product could be switched by merely changing Selectfluor for fluoroiodane.

In summary, we have developed efficient protocols for accessing two new classes of fluorinated heterocycles using fluoroiodane reagent $\mathbf{1}$. Alternative selectivity to that displayed by electrophilic fluorinating reagents (e.g. Selectfluor) is clearly demonstrated and thus, this study shows that fluoroiodane 1 is a complementary reagent for diversifying fluorination methodology. Mechanochemistry has provided a dramatic improvement in the yield and selectivity to fluorinated dihydrooxazines, and in so doing has highlighted that mechanistically complex reactions can be readily achieved in a ball-mill.

\section{Conflicts of interest}

There are no conflicts to declare.

\section{Notes and references}

1 (a) B. G. de la Torre and F. Albericio, Molecules, 2020, 25, 745; (b) F. Lovering, J. Bikker and C. Humblet, J. Med. Chem., 2009, 52, 6752; (c) S. Purser, P. R. Moore, S. Swallow and V. Gouverneur, Chem. Soc. Rev., 2008, 37, 320.

2 J. Zhao, M. Jiang and J.-T. Liu, Org. Chem. Front., 2018, 5, 1155.

3 (a) Y.-Y. Liu, J. Yang, R.-J. Song and J.-H. Li, Adv. Synth. Catal., 2014, 356, 2913; (b) J. Zhao, M. Jiang and J.-T. Liu, Adv. Synth. Catal., 2017, 359, 1626; (c) W. Kong, Q. Guo, Z. Xu, G. Wang, X. Jiang and R. Wang, Org. Lett., 2015, 17, 3686.

4 (a) C. Y. Legault and J. Prévost, Acta Crystallogr., Sect. E: Crystallogr. Commun., 2012, 68, o1238; (b) V. Matoušek, E. Pietrasiak, R. Schwenk and A. Togni, J. Org. Chem., 2013, 78, 6763; (c) G. C. Geary, E. G. Hope, K. Singh and A. M. Stuart, Chem. Commun., 2013, 49, 9263.

5 (a) N. O. Ilchenko, B. O. A. Tasch and K. J. Szabó, Angew. Chem., Int. Ed., 2014, 53, 12897; (b) G. C. Geary, E. G. Hope, K. Singh and A. M. Stuart, $R S C A d v ., 2015,5,16501 ;(c)$ W. Yuan and K. J. Szabó, Angew. Chem., Int. Ed., 2015, 54, 8533; (d) A. Ulmer, C. Brunner, A. M. Arnold, A. Pöthig and T. Gulder, Chem. - Eur. J., 2016, 22, 3660; (e) W. Yuan, L. Eriksson and K. J. Szabó, Angew. Chem., Int. Ed., 2016, 55, 8410; $(f)$ N. O. Ilchenko, M. Hedberg and K. J. Szabó, Chem. Sci., 2017, 8, 1056; $(g)$ B. Xing, C. Ni and J. Hu, Angew. Chem., Int. Ed., 2018, 57, 9896; (h) A. M. H. Abudken, E. G. Hope, K. Singh and A. M. Stuart, Org. Biomol. Chem., 2020, 18, 6140; (i) S. Yang, S. Shi, Y. Chen and Z. Ding, J. Org. Chem., 2021, 86, DOI: 10.1021/ acs.joc.1c00159.

6 (a) G. C. Geary, E. G. Hope and A. M. Stuart, Angew. Chem., Int. Ed., 2015, 54, 14911; (b) H. K. Minhas, W. Riley, A. M. Stuart and M. Urbonaite, Org. Biomol. Chem., 2018, 16, 7170.

7 (a) J. L. Howard, Q. Cao and D. L. Browne, Chem. Sci., 2018, 9, 3080; (b) G.-W. Wang, Chem. Soc. Rev., 2013, 42, 7668; (c) A. Stolle, T. Szuppa, S. E. S. Leonhardt and B. Ondruschka, Chem. Soc. Rev., 2011, 40, 2317; (d) T. Friščić, C. Mottillo and H. M. Titi, Angew. Chem., Int. Ed., 2020, 59, 1018; (e) J. G. Hernández and C. Bolm, J. Org. Chem., 2017, 82, 4007; $(f)$ A. Porcheddu, E. Colacino, L. De Luca and F. Delogu, ACS Catal., 2020, 10, 8344; $(g)$ J. Andersen and J. Mack, Green Chem., 2018, 20, 1435; (h) S. L. James, et al., Chem. Soc. Rev., 2012, 41, 413; (i) M. Leonardi, M. Villacampa and J. C. Menéndez, Chem. Sci., 2018, 9, 2042; $(j)$ T.-X. Métro, J. Martinez and F. Lamaty, ACS Sustainable Chem. Eng., 2017, 5, 9599.

$8 \mathrm{~J}$. Wong and Y.-Y. Yeung, RSC Adv., 2021, 11, 13564.

9 (a) J. L. Howard, Y. Sagatov, L. Repusseau, C. Schotten and D. L. Browne, Green Chem., 2017, 19, 2798; (b) J. L. Howard, Y. Sagatov and D. L. Browne, Tetrahedron, 2018, 74, 3118; (c) Y. Wang, H. Wang, Y. Jiang, C. Zhang, J. Shao and D. Xu, Green Chem., 2017, 19, 1674.

10 (a) J. L. Howard, W. Nicholson, Y. Sagatov and D. L. Browne, Beilstein J. Org. Chem., 2017, 13, 1950; (b) D. Krištofíková, M. Mečiarová, E. Rakovský and R. Šebesta, ACS Sustainable Chem. Eng., 2020, 8, 14417.

11 M. Sawaguchi, S. Hara, T. Fukuhara and N. Yoneda, J. Fluorine Chem., 2000, 104, 277.

12 For ring expansion of cyclic ethers with $p$-iodotoluene difluoride see: T. Inagaki, Y. Nakamura, M. Sawaguchi, N. Yoneda, S. Ayuba and S. Hara, Tetrahedron Lett., 2003, 44, 4117. 